\title{
IN VITRO STUDY OF BACTERICIDAL EFFECT OF ANTIMICROBIAL AGENTS USED IN MODERN ENDODONTICS
}

\author{
V. Kalchinov*, Sl. Dimitrov*, M. Belcheva** \\ * Department of Conservative Dentistry \\ ** Department of Image and Oral Diagnostic \\ Faculty of Dental Medicine, Medical University - Sofia, Bulgaria
}

\section{SUMMARY:}

The purpose of this study is to carry out comparative in vitro study of bactericidal effect of antimicrobial drugs used in modern endodontics against some basic microbial agents of periodontitis. Using the agar diffusion method (experiment with sterilized disks and wells) antimicrobial effect of five antimicrobial agents used in endodontics is evaluated: Rockle, Cresophene, $\mathrm{I}_{2} / \mathrm{KI}$, Cupral, $\mathrm{Ca}(\mathrm{OH})_{2}$ - high dispersion. For the study three types of nutritious media are used: BHI- agar, MH- agar, Columbi blood agar. Tested microorganisms are: E. faecalis, S. aureus, bacterium of Prevotella spp. We found out through the standard agar diffusion method with disks that $\mathrm{I}_{2} / \mathrm{KI}$ has biggest bactericidal effect except for two cases when Cresophene is present. Using the method with wells we studied the antimicrobial effect only for Cupral and $\mathrm{Ca}(\mathrm{OH})_{2}$ - high dispersion. These agents have almost the same antimicrobial activity except for two cases when Cupral has bigger antimicrobial activity.

Key words: endodontics microbiology, agar diffusion method

\section{INTRODUCTION:}

Lots of authors consider microorganisms for primary etiologic agents in endodontics diseases(6,7,8). Microbial flora in the root canals when a treatment is failed consists mainly of limited number of anaerobes. They thrive in an environment that lacks oxygen and contains a limited amount of nutrients (7). Bacteria may still be detected in the root canal system after chemomechanical preparation. Their numbers may rapidly increase in the empty canals in the period between appointments (9). Numerous studies have reported that bacteria may remain viable in ramifications, isthmian and dentinal tubules of the root canal system even after complete chemomechanical preparation $(3,9)$.

According to Yu-heng Lin 2003 (6) the rate of successful retreatment at infected root canals with Gramnegative culture is $80 \%$. Root canals with Gram- positive culture have only $33 \%$ success rate. The same author discovered numerous species of anaerobic bacteria in teeth with failed endodontic treatment. Some of these bacteria include E.faecalis, S.anginosus, B.gracilis, F.nucleatum (7). E.faecalis was found to be the most prevalent bacteria in failed root canal system $(6,7)$.

The general task in endodontic treatment of infected root canals is destruction and complete elimination of microorganisms and protection from reinfection.

This aim is accomplished by using mainly antimicrobial agents through different methods: irrigation, application, ultrasonic preparation of root canals, iontophoresis. Intracanal dressing with drugs must have wide antibacterial spectrum and time for action on various polimicrobial infection (5). Also, this intracanal medication has to serve as mechanical barrier by reducing marginal infiltration, providing canal dryness, neutralizing tissue debris and decreasing periapical tissue inflammation.

Antibacterial agents used most often in infected root canals are: phenol compounds, chlorhexidine (depo, solution, gel), $\mathrm{I}_{2} / \mathrm{KI}, \mathrm{Ca}(\mathrm{OH})_{2}$, Cupral, proteolitic enzyms, antibiotics, sulfonamids.

Calcium hydroxide $\mathrm{Ca}(\mathrm{OH})_{2}$ is a drug widely used as an intracannal dressing between the appointments in endodontic therapy and possesses significant antibacterial properties $(4,8)$. It is used in composition of pastes and cements. These agents possess antibacterial, antiinflammatory and dryness effect and have very good biological activity (4). These properties are due to the ionic dissociation into $\mathrm{Ca}^{2+}$ and $\mathrm{OH}^{-}$ions $(8,9)$. Viscous and oily vehicles promote continuous slow diffusion of ions with low solubility (8). These agents except for antibacterial properties possess anti-inflammatory properties primarily due to the high $\mathrm{pH}$ value 12.5 in the surrounding environment after their dissolution. Most bacteria cannot resist a pH over 9.5. Calcium hydroxide also has some drawbacks: it induces pulp necrosis and inflammation over the first few days, it has poor hardening, it is easily dissolve. These properties do not allow tight filling of root canals. This determine their application as temporary material for intracanal dressing (4).

Cupral - this agent possesses powerful bactericidal 
effect, it represents heterogeneous stabilized balance system. It contains negative charged substances: a) anion on the base of copper hydroxide $\left[\mathrm{Cu}(\mathrm{OH})_{4}\right]^{2-}$, b) nanosphere of copper hydroxide, negative charged c) $\mathrm{OH}^{-}$anions. Negative charged substances are led to depth $500 \mathrm{~mm}$ in dentinal tubules by direct current, while $\mathrm{Ca}(\mathrm{OH})_{2}$ is led in root canal with application, depth of penetration of $\mathrm{OH}^{-}$ anions in dentinal tubules is $200 \mathrm{Mm}$ (2).

$\mathrm{I}_{2} / \mathrm{KI}$ - this drug have powerful antimicrobial effect. It is consist of iodine and iodine ions. These ions are led in root canals by direct current (1).

Rockle - antimicrobial agent for treatment of infected root canals. It is led with application. Chemical composition is: Dexamethasone acetate, Phenol, Gaiacol (new formula).

Cresophene - antimicrobial agent for treatment of infected root canals. It is led with application. Chemical composition is: Dexamethasone acetate, Thymol (new formula).

Antimicrobial activity of endodontic materials can be evaluated either in vitro or in vivo. Agar diffusion method, despite its disadvantages such as: lack of standardization of inoclum density, adequate culture medium, agar viscosity, plate storage conditions, size and number of specimens per plate, time and temperature of incubation, is still the most widely used in vitro method of evaluation of antimicrobial activity (5). Numerous studies on bactericidal effect of antimicrobial agents in endodontics are contradictory and incomplete.

\section{AIM:}

In the present study we put the task to carry out comparative in vitro study of antimicrobial effect of the most often used agents in endodontics against some basic microbial agents of periodontitis by agar diffusion method.

\section{MATERIALS AND METHODS:}

Using the agar diffusion method antimicrobial effect of antimicrobial agents used in endodontics is evaluated. The agar diffusion method is accomplished either with sterilized disks, or with wells.

1. When disks are used (as for antibiograms), they are soaked in advance with drugs, after that the disks are put on agar surface $(6,7,8)$. (photo No. 1 )

2. When the second method is used, the wells are excavated in agar substance with puncher - diameter $6 \mathrm{~mm}$ and are filled with antimicrobial agents $(5,9,3)$. (photo No. 2)

The antimicrobial effect of five drugs is explored:
Rockle, Cresophene, $\mathrm{I}_{2} / \mathrm{KI}, \mathrm{Ca}(\mathrm{OH})_{2}$ - high dispersion, Cupral with most common application in endodontics on the following test microorganisms: Enterococcus faecalis, Staphylococcus aureus, species of Prevotella spp. Nutritious medium which are necessary for these bacterial species are: Brain Heart Infusion agar (BHI), Muller Hinton agar $(\mathrm{MH})$, Columbia blood agar.

When tested microorganisms are E.faecalis and $S$. aureus and agar diffusion method is implemented with disks, for each bacterial strain 12 petri plates are needed . Six petri plates are with $\mathrm{BHI}$-agar and six with $\mathrm{MH}$-agar. In each petri plate there are five sterilized disks soaked with antimicrobial agents- Rockle, Cresophene, I2/KI, Cupral, $\mathrm{Ca}(\mathrm{OH}) 2$. When tested microorganisms are the same but agar diffusion method is implemented with wells, for each bacterial strains 12 petri plates v. Six petri plates are with BHI- agar and six with $\mathrm{MH}$-agar. In each petri plate there are two wells. The wells are filled with suspension of Cupral or suspension of $\mathrm{Ca}(\mathrm{OH})_{2}$ - high dispersion.

In our study when tested microorganism was of Prevotella spp. we used only one nutritious medium: Columbia blood agar. For agar diffusion method with disks are necessary six petri plates. In each petri plate there are five disks soaked with the same drugs as when tested microorganism are E.faecalis or S.aureus. For agar diffusion method with wells are necessary six petri plates. In each petri plate there are two wells. The wells are filled with suspension of Cupral or suspension of $\mathrm{Ca}(\mathrm{OH})_{2}$ - high dispersion.

The microorganism suspensions are adjusted to tube 0.5 of the Mac Farland scale. Bacterial inoculum is spread on agar surface. After the antimicrobial agents are put in agar, petri plates are kept at room temperature for 2 hours for prediffusion of materials. After that petri plates with $S$. aureus and E.faecalis are cultivated for 24 hours in aerobic conditions. Bactericidal effect of drugs is assessed by measuring the diameters (in $\mathrm{mm}$ ) of inhibition haloes around disks and wells. When tested microorganism is of Prevotella spp bacterial suspension is adjusted to tube 0.5 of the MacFarland scale, too. After the antimicrobial agents are put in agar, the petri plates are placed immediately in microanaerostat for anaerobic cultivation for 2 days.

\section{RESULTS:}

On tables 1- 4 the results from the study are shown. The bactericidal effect of five drugs on tested bacteria is explored. 
Table 1. Experiment - Disks

\begin{tabular}{|c|c|c|c|c|c|}
\hline \multirow{2}{*}{ Microorganism } & \multirow{2}{*}{ Nutritious medium } & \multirow{2}{*}{$\begin{array}{l}\text { Antimicrobial } \\
\text { agent }\end{array}$} & \multicolumn{3}{|c|}{ Persentiles } \\
\hline & & & Median & 25 th & 75 th \\
\hline \multirow{10}{*}{ E. faecalis } & \multirow{5}{*}{ BHI-agar } & $\mathrm{Ca}(\mathrm{OH})_{2}$ & 6.5 & 6 & 7 \\
\hline & & Cupral & 6.5 & 6 & 7 \\
\hline & & Rockle & 10 & 9.5 & 10.5 \\
\hline & & Cresophene & 17.5 & 15.75 & 18.25 \\
\hline & & $\mathrm{I}_{2 / \mathrm{KI}}$ & 19 & 17 & 21.25 \\
\hline & \multirow{5}{*}{ MH-agar } & $\mathrm{Ca}(\mathrm{OH})_{2}$ & 6 & 6 & 6 \\
\hline & & Cupral & 6 & 6 & 6 \\
\hline & & Rockle & 9 & 8.75 & 9 \\
\hline & & Cresophene & 13 & 11.75 & 13.5 \\
\hline & & $\mathrm{I}_{2 / \mathrm{KI}}$ & 22 & 19.5 & 25 \\
\hline \multirow{10}{*}{ S. aureus } & \multirow{5}{*}{ BHI-agar } & $\mathrm{Ca}(\mathrm{OH})_{2}$ & 7 & 6 & 7.75 \\
\hline & & Cupral & 9.5 & 8.5 & 10 \\
\hline & & Rockle & 21.5 & 19.25 & 35 \\
\hline & & $\mathrm{I}_{2 / \mathrm{KI}}$ & 23.5 & 16.75 & 34.5 \\
\hline & & Cresophene & 37 & 32.25 & 46 \\
\hline & \multirow{5}{*}{ MH-agar } & Cupral & 9.5 & 7 & 12.25 \\
\hline & & $\mathrm{Ca}(\mathrm{OH})_{2}$ & 11 & 9.75 & 13.5 \\
\hline & & Rockle & 26 & 19.5 & 32.5 \\
\hline & & Cresophene & 28.5 & 25 & 37 \\
\hline & & $\mathrm{I}_{2 / \mathrm{KI}}$ & 33.5 & 27.75 & 42 \\
\hline \multirow{5}{*}{ Prevotella spp } & \multirow{5}{*}{ Columbia blood agar } & $\mathrm{Ca}(\mathrm{OH})_{2}$ & 10 & 9 & 12.5 \\
\hline & & Rockle & 24 & 19.5 & 38.5 \\
\hline & & $\mathrm{I}_{2} / \mathrm{KI}$ & 27 & 24.5 & 28 \\
\hline & & Cupral & 32.5 & 28.5 & 36.25 \\
\hline & & Cresophene & 36 & 33.5 & 38 \\
\hline
\end{tabular}

On tables 1,2 the results from bactericidal effect of separate antimicrobial agents on given microorganism at respective nutritious medium (a unit of measurement of 50-th persentiles /mediana/, 25-th and 75-th persentiles is millimeter) are shown. The results are arranged in crescent series depending on effect (arrangement is according to median). The bigger value of median indicates bigger diameter (in $\mathrm{mm}$ ) of zone of inhibition of bacterial growth around the disk or the well.

Table 2. Experiment- Wells

\begin{tabular}{|c|c|c|c|c|c|}
\hline \multirow{2}{*}{ Microorganism } & \multirow{2}{*}{ Nutritious medium } & \multirow{2}{*}{$\begin{array}{l}\text { Antimicrobial } \\
\text { agent }\end{array}$} & \multicolumn{3}{|c|}{ Percentiles } \\
\hline & & & Median & $25 t h$ & $75 \mathrm{th}$ \\
\hline \multirow{4}{*}{ E. faecalis } & \multirow{2}{*}{ BHI-agar } & $\mathrm{Ca}(\mathrm{OH})_{2}$ & 8.5 & 7 & 9 \\
\hline & & Cupral & 7.5 & 7 & 8.75 \\
\hline & \multirow{2}{*}{ MH-agar } & $\mathrm{Ca}(\mathrm{OH})_{2}$ & 8.5 & 7.75 & 9 \\
\hline & & Cupral & 8 & 8 & 8.25 \\
\hline \multirow[b]{2}{*}{ S. aureus } & \multirow{2}{*}{ BHI-agar } & $\mathrm{Ca}(\mathrm{OH})_{2}$ & 10 & 9.5 & 11 \\
\hline & & Cupral & 11.5 & 11 & 12.25 \\
\hline
\end{tabular}




\begin{tabular}{|c|l|l|l|c|c|}
\hline & \multirow{2}{*}{ MH-agar } & $\mathrm{Ca}(\mathrm{OH})_{2}$ & 15 & 14 & 15.25 \\
\cline { 3 - 6 } & & $\mathrm{Cupral}$ & 15 & 14 & 15 \\
\hline \multirow{2}{*}{ Prevotella spp } & \multirow{2}{*}{ Columbia blood agar } & $\mathrm{Ca}(\mathrm{OH})_{2}$ & 21 & 20 & 22.75 \\
\cline { 3 - 6 } & & Cupral & 32 & 30 & 32.5 \\
\hline
\end{tabular}

The results from carried out studies using the standard agar diffusion method (experiment with disks and wells)are shown on the statistical tables 3,4. The data is a result of the non-parametric tests Kruskal-Wallis and Mann-Whitney.

Table 3. Test for comparing results of bactericidal action of antimicrobial agents (Rockle, Cresophene, $\mathrm{I}_{2} / \mathrm{KI}, \mathrm{Ca}(\mathrm{OH})_{2}$, Cupral) Experiment - Disks

\begin{tabular}{|l|l|c|c|c|}
\hline \multicolumn{4}{|c|}{ Non- parametric test (Kruskal-Wallis) } \\
\hline Microorganism & Nutritious medium & $\chi 2$ & $d f$ & $p$ \\
\hline S. aureus & BHI-agar & 23.678 & 4 & $0.000^{*}$ \\
\hline S. aureus & MH-agar & 22.571 & 4 & $0.000^{*}$ \\
\hline E. faecalis & BHI-agar & 25.946 & 4 & $0.000^{*}$ \\
\hline E. faecalis & MH-agar & 28.439 & 4 & $0.000^{*}$ \\
\hline Prevotella spp & Columbia_blood_agar & 17.194 & 4 & $0.002^{*}$ \\
\hline
\end{tabular}

Table 4. Test for comparing results of bactericidal action of antimicrobial agents $\left(\mathrm{Ca}(\mathrm{OH})_{2}\right.$, Cupral) Experiment- Wells

\begin{tabular}{|l|l|r|r|}
\hline \multicolumn{4}{|l|}{ Non- parametric test (Mann-Whitney) } \\
\hline Microorganism & Nutritious medium & $U$ & \multicolumn{1}{c|}{$p$} \\
\hline S.aureus & BHI-agar & 3 & $0.015^{*}$ \\
\hline S.aureus & MH-agar & 16 & 0.818 \\
\hline E.faecalis & BHI-agar & 14 & 0.589 \\
\hline E.faecalis & MH-agar & 14.5 & 0.589 \\
\hline Prevotella spp & Columbia_blood_agar & 0 & $0.002^{*}$ \\
\hline
\end{tabular}

*- the star indicates that there is statistical significant difference in bactericidal action of separate antimicrobial agents $(p<0.05)$. In the other cases $(p>0.05)$ there is no statistical significant difference in bactericidal action of separate antimicrobial agents.

\section{DISCUSSION:}

The agar diffusion method is one of the most often used methods for antimicrobial activity assessment. Several factors are important for diffusion capacity of materials in agar: contact between material and agar, molecular weight, shape of the antimicrobial agent, concentration of test material, agar gel viscosity and etc. Furthermore, control and standardization of inoculation density, evaluation of results, selection of agar medium, incubation temperature of plates, and reading point of inhibition haloes are also restricting factors affecting the dynamics and variability of diffusion tests in an agar medium (5). Facultatative anaerobic and strict anaerobic microorganisms were used for this study.

When the agar diffusion method with disks is used and the tested microorganism is E.faecalis $\mathrm{I}_{2} / \mathrm{KI}$ followed by Cresophene, Rockle, Cupral, $\mathrm{Ca}(\mathrm{OH})_{2}$ has the biggest bactericidal effect. When tested microorganism is S.aureus ( nutritious medium is BHI-agar) Cresophene followed by $\mathrm{I}_{2} / \mathrm{KI}$, Rockle, Cupral, $\mathrm{Ca}(\mathrm{OH})_{2}$ has the biggest bactericidal effect. If nutritious medium is $\mathrm{MH}$-agar $\mathrm{I}_{2} / \mathrm{KI}$ followed by Cresophene, Rockle, $\mathrm{Ca}(\mathrm{OH})_{2}$, Cupral has the biggest bactericidal effect. When tested microorganism is of Prevotella spp, Cupral has commensurable antimicrobial activity with Cresophene. Here antimicrobial agent with biggest bactericidal effect is Cresophene next is Cupral, $\mathrm{I}_{2} /$ $\mathrm{KI}$, Rockle, $\mathrm{Ca}(\mathrm{OH})_{2}$. On table 2 the results for bactericidal effect of Cupral and $\mathrm{Ca}(\mathrm{OH})_{2}$ (experiment with wells) are shown. These antimicrobial agents have commensurable antimicrobial activity with the exception of two cases, when test bacterium is from Prevotella spp (Cupral- $32 \mathrm{~mm}$, $\mathrm{Ca}(\mathrm{OH})_{2}-21 \mathrm{~mm}$ inhibition haloes), and S.aureus ( BHI-agar, Cupral $\left.-11.5 \mathrm{~mm}, \mathrm{Ca}(\mathrm{OH})_{2}-10 \mathrm{~mm}\right)$. In these two cases Cupral has bigger antimicrobial activity than $\mathrm{Ca}(\mathrm{OH})_{2}$. E.faecalis is most resistant to the influence of the same agents. Analysis of the data shown on table 1 indicates, that bactericidal effect of the explored five agents is different towards the three tested bacteria. Cresophene and Cupral possess the most powerful antibacterial effect against bacterium of Prevotella spp ( 36 and $32.5 \mathrm{~mm}$ inhibition haloes). $\mathrm{I}_{2} / \mathrm{KI}$ and Cresophene possess the most powerful antibacterial effect against S.aureus $\left(\mathrm{I}_{2} / \mathrm{KI}-33.5 \mathrm{~mm}\right.$, Cresophene-28.5 mm in MH agar; Cresophene $-37 \mathrm{~mm}, \mathrm{I}_{2} /$ KI- $23.5 \mathrm{~mm}$ in BHI agar). The five antimicrobial agents have the weakest action against $E$.faecalis. 


\section{CONCLUSION:}

The five antimicrobial agents have different action towards tested bacteria. Cresophene and Cupral possess the most powerful antibacterial effect against bacterium of Prevotella spp and $\mathrm{I}_{2} / \mathrm{KI}$, Cresophene against S.aureus.
E.faecalis is the most resistant bacterium in this study. Cresophene makes the growth of E.faecalis about three times weaker in comparison with Prevotella spp and about two times weaker than S.aureus.
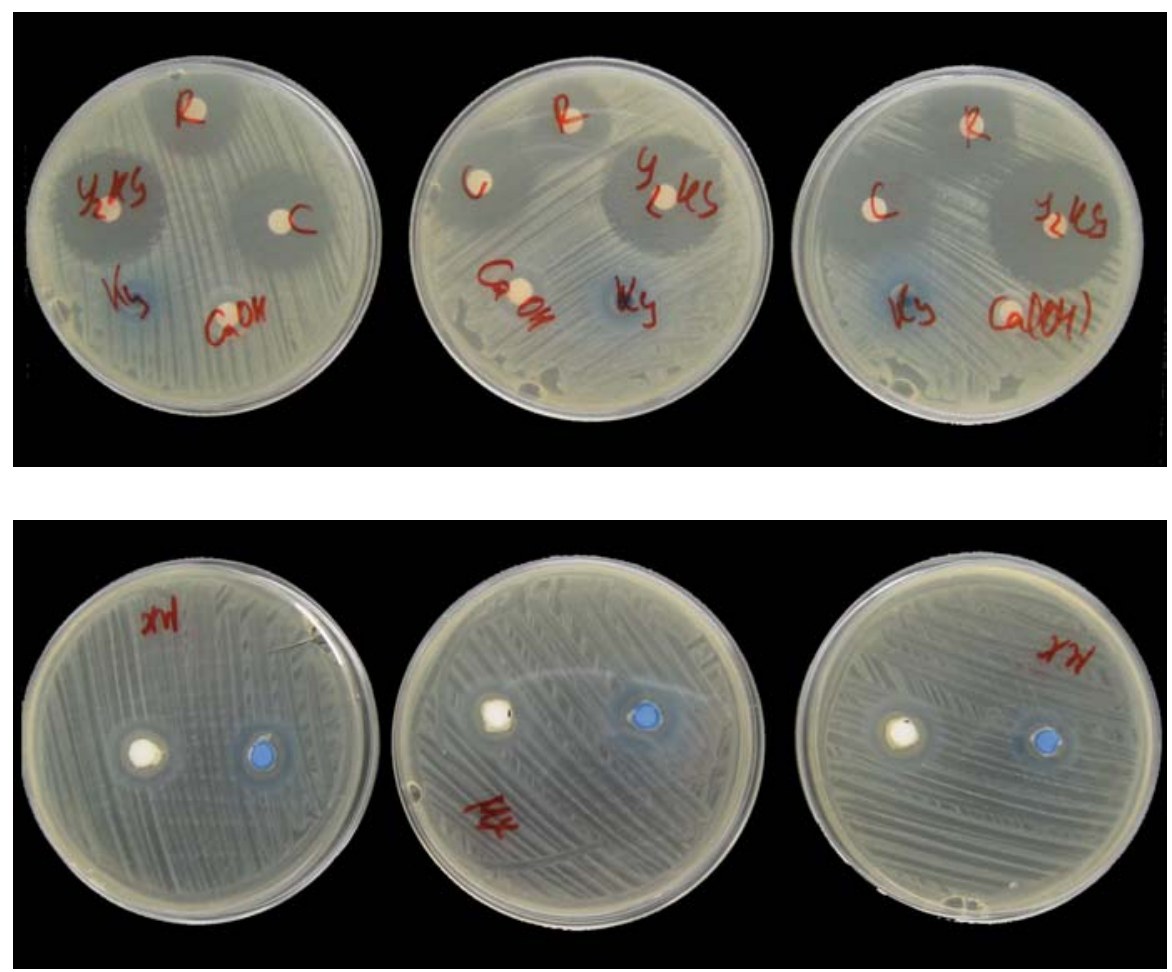

Photo No. 1.

\section{Photo No. 2.}

Acknowledgments: This study was sponsored by Grant № 21-D/2008 from Medicine University- Sofia. We sincerely thank assoc.prof. Bojanova (Faculty of Medicine -Sofia , Department of Medicine Microbiology) for placing the bacterium of Prevotella spp at our disposal.

\section{REFERENCES:}

1. Балчева Е. Н. и кол. 3: Физиотерапия при стоматологичните заболявания, Клиника на терапевтичната стоматология, гр. София, изд. Медицина и физкултура, 1999

2. Садовский В.В. ДЕПОФОРЕЗ теоретическо обоснование и клиническо применение гр. Москва, изд. МЕДИЦИНСКАЯ КНИГА, 2006г, 45 стр, 1-45

3. Cobankara F.K. et al. In vitro antibacterial activities of root-canal sealers by using two different methods. Journal of Endod. Jan. 2004, vol.30, №1, 57-60

4. Kouassi M. et al Antibacterial effect of hydraulic calcium phosphate cement for dental applications. Journal of Endod. Feb. 2003, vol.29, №2, 100-103

5. Leonardo M. R. et al. In vitro evaluation of antimicrobial activity of sealers and pastes used in endodontics. Journal of Endod. July 2000, vol.26, №7, 391-394

6. Lin Y., A.K. Mickel, S. Chogle Effectiveness of selected materials against Enterococcus faecalis: Part 3. The antibacterial effect of calcium hydroxide and chlorhexidine on Enterococcus faecalis. Journal of Endod. Sept.2003, vol. 29, №

\section{$9,565-566$}

7. Mickel A.K., T.H. Nguyen, S. Chogle Antimicrobial activity of endodontic sealers on Enterococcus faecalis. Journal of Endod. April 2003, vol.29, №4, 257-258

8. Mickel A.K., P. Sharma, S. Chogle Effectiveness of stannous fluoride and calcium hydroxide against Enterococcus faecalis. Journal of Endod. April 2003, vol. 29, № 4, 259-260

9. Morrier J.J. et al. Antimicrobial activity of $\mathrm{Ca}(\mathrm{OH})_{2}$ dental cements: an in vitro study. Journal of Endod. Jan.2003, vol. 29, № 1, 51-54

Address for correspondence:

Vasko Kalchinov

Faculty of Dental Medicine - Sofia , Department of Conservative Dentistry,

1, George Sofiiski str., Sofia, mob. tel.: +359/898 7647 95, e-mail: vasko_kalchinov@mail.bg 